\title{
Hepatitis C Virus
}

National Cancer Institute

\section{Source}

National Cancer Institute. Hepatitis C Virus. NCI Thesaurus. Code C14312.

A small, enveloped, positive sense single strand RNA virus in the family Flaviviridae. The genome consists of a single RNA molecule with a single open reading frame. 